\title{
Cumulative improbability
}

\author{
Timothy F. Christian, MD, MPA ${ }^{\text {a }}$ \\ a Research Department, Cardiac Imaging, DeMatteis Research Center, St Francis Hospital, \\ Roslyn, NY
}

Received Jun 16, 2017; accepted Jun 16, 2017

doi: 10.1007/s12350-017-1027-4

Few things in cardiology seem as intuitive as the logic for measuring myocardial dyssynchrony by imaging. And yet the ability to show an imaging strategy benefit has been inconclusive. The issues for imaging as a decision tool for cardiac resynchronization therapy (CRT) are clearly laid out by the two accompanying position papers. ${ }^{1,2}$ In addition to these, there are some unique aspects of this field which greatly expand the variability in evaluating the effectiveness of biventricular pacing. As these accumulate, the ability to measure the true impact of CRT on patient outcome becomes obscured. An analogy is the cumulative drop in flow when multiple resistors are arranged in series, rather than in parallel. In a serial configuration, resistance is an additive sum. When significant variability is present for each step of an evaluation pathway, the additive effect can be overwhelming. This analogy is applicable to the question of whether or not gated SPECT can predict a response CRT with concurrent right and left ventricular pacing. Each step in the CRT pathway adds, in a unique manner, a degree of uncertainty that clouds the ability to assess an imaging strategy.

The Many Pathways to CHF The end-product of ventricular dyssynchrony can be arrived at from multiple etiologies including ischemic heart disease, myocarditis, cardiomyopathy and conduction tissue degeneration. Each of these has a different prognosis independent of any pacing strategy. Yet they are grouped together when considering outcomes to CRT. This introduces a layer of variability at the outset that will be independent of any imaging strategy to define a response to CRT. It is unreasonable to expect that these separate diseases will respond uniformly to a therapy nor will they share a similar prognosis. Consequently, the patient population

Reprint requests: Timothy F. Christian, MD, MPA, Research Department, Cardiac Imaging, DeMatteis Research Center, St Francis Hospital, Roslyn, NY; timothy.christian@rutgers.edu

J Nucl Cardiol 2018;25:536-9

$1071-3581 / \$ 34.00$

Copyright (c) 2017 American Society of Nuclear Cardiology. is heterogeneous. This variability has been accounted for in randomized trials of CRT therapy. Models accounting for clinical variables and co-morbidities have shown many clinical factors which are independent predictors of the response to CRT. ${ }^{3,4}$ These models have worked fairly well. Failure to account for them will impact any evaluation of effectiveness. This also suggests that most of the information concerning the response to CRT is in the clinical level, leaving less room for imaging to provide an independent contribution. This was the case for echocardiography in the major imaging trial for CRT. ${ }^{5}$

The Physics of SPECT The definition of asynchrony by ECG criteria has varied. Initially it was described as a QRS widening $>120 \mathrm{msec}$. More recently it has been lengthened to $150 \mathrm{msec}$, which simply reveals the problems associated with dichotomizing a continuous variable into a disease definition. This implies that milliseconds matter. Gated SPECT imaging is among the worst performers when it comes to temporal resolution. Assuming a 8 frame series of images per R-R interval at a heart rate of 75 , the temporal resolution of each frame is $100 \mathrm{~ms}$. At slower heart rates, the temporal resolution lengthens further. M-mode echocardiography has the best temporal resolution at 1$2 \mathrm{~ms}$. For an event that requires fine differentiation in time, gated SPECT is a blunt instrument.

The other physical constraint of SPECT is spatial resolution. The spatial resolution of SPECT is about $10 \mathrm{~mm}$. This is often larger than the thickness of the ventricular wall which makes motion tracking on the $\mathrm{mm}$ level problematic. Consequently, investigators have used a counts-based approach focusing on brightening of the wall. The two resolution confounders together are likely additive in decreasing the ability to detect dyssynchrony at $120 \mathrm{~ms}$. Both of these physical parameters introduce a degree of variability for the assessment of CRT as a strategy.

LV Lead Placement The success of CRT is, in part, due to the skill and technique of the therapy. Unlike a randomized trial where the therapy is definitive, such as 
taking a pill or placebo, the therapy of CRT is variable. The variability is multifactorial and includes coronary sinus anatomy, presence of infarction or fibrosis in the tissue that is in contact with the lead, arrhythmias, and issues with pacer programming. It is difficult to know the amount of variability introduced at the intervention level. But clearly the potential for uncertainty about therapy effectiveness is present.

Follow-up Echocardiography One outcome measure for CRT is a change in volume and ventricular performance. The confidence limits for echocardiographic volumes derived by 2D echo without contrast are fairly wide under good conditions. ${ }^{6}$ Patients with heart failure often have other co-morbidities that make imaging by echo challenging. While temporal and spatial resolution are excellent, contrast to noise is not. Consequently, identification of endocardial surfaces can be inaccurate and varies by anatomic location. Small errors in edge detection can produce misleading volume measures as the error is multiplied for echo derived volume formulas. This adds a level of variability in an important outcome metric.

Clinical End-points The subjective assessment of improvement in symptoms can be quite variable as well. More quantitative approaches such as the 6-minute walk test also have a degree of uncertainty concerning reproducibility. ${ }^{7}$ Consequently, what determines patient benefit, aside from hard end-points such as death, is also subject to variability.

Serial Cumulative Variability In Nuclear cardiology, Bayesian principles dominate. That is to say that clinical decision making progresses from lesser to greater certainty as to the probability of disease with the serial use of our tools. In the usual scenario, a patient with chest pain presents to a caretakers office. Before the caretaker walks into the room, the probability of the presence of significant coronary artery disease and potential myocardial infarction is $50 \%$. Upon meeting the patient and taking a history that probability either increases or decreases based on the answers and findings to well established risk factors. There is a confidence interval that tightens around a probability of disease with new each new piece of data, provided that the source of the data provides new and reliable information, termed incremental value. Importantly, each new piece of data builds on those discovered before it. In this manner, the new probability is a product of the prior data plus the new piece. As cardiologists, we build the case for or against disease serially by taking a history and physical, perform ECG stress testing, then perfusion imaging. Each step builds upon the prior and is progressively more accurate (Figure 1).

CRT does not lend itself well to this approach. Instead of getting new and more accurate information with each step, there is increased uncertainty. The differing etiologies for the CRT population introduce the potential for disparate responses to therapy. Consequently, a level of variability is introduced from the outset. Each progressive step: the physics of imaging, lead placement, volume metrics, and clinical outcomes disperse our knowledge of the probability of the effectiveness of CRT (Figure 2). This variability is in series and, therefore, cumulative.

For example, compare two patients, both with identical gated SPECT studies showing wide phase dispersion and low LVEF. Patient \#1 has Class III CHF, a history of dilated cardiomyopathy, LBBB, no obstructive $\mathrm{CAD}$ and minimal myocardial fibrosis by CMRI. The patient has uneventful CRT with improvement in CHF class and a 10 point increase in LVEF by follow-up echocardiography. Patient \#2 has Class III $\mathrm{CHF}$, ischemic cardiomyopathy $\mathrm{s} / \mathrm{p}$ anterior and inferolateral MI, LBBB, and extensive scarring by CMRI. CRT is performed with difficulty due to high pacing thresholds over the coronary sinus. Ventricular volumes are hard to assess by follow-up echocardiography due to poor acoustic windows. There is minimal clinical improvement and the patient dies 30 days later from an ischemic event. While the gated SPECT scan predicted success in both cases, the outcome is blurred by the gathering levels of uncertainty and confounders.

The Bottom Line Because of the cumulative uncertainty present in the CRT evaluation algorithm, it is a stretch to promote gated SPECT to predict patient success, despite the strong arguments made in support of the technique. ${ }^{1}$ In addition to cumulative uncertainty, a major concern is the relative failure of echocardiography in clinical trials to provide accurate predictions. Echocardiography is superior in terms of the physics required to measure contractile timing issues. If this modality does not work, gated SPECT, with all its physical limitations, is unlikely to do better. Because of the strength of clinical predictors for CRT benefit, imaging may be limited to any additional independent information provided. However, the ability to concurrently assess viability in patients with ischemic heart disease being considered for CRT may be an application for gated SPECT if it is used to identify prospectively the success of LV pacing. MRI, however, has better physics for this assessment. Nuclear cardiology has had remarkable gains in both technology and acceptance over the last 30 years. This goes back to the early modalities of planar thallium imaging and radionuclide ventriculography. Part of that success has been the selfcritical nature of the field, which sometimes has been carried to a fault. But it has kept the field credible. We should be cautious about straying into fields where the physics are against us. 


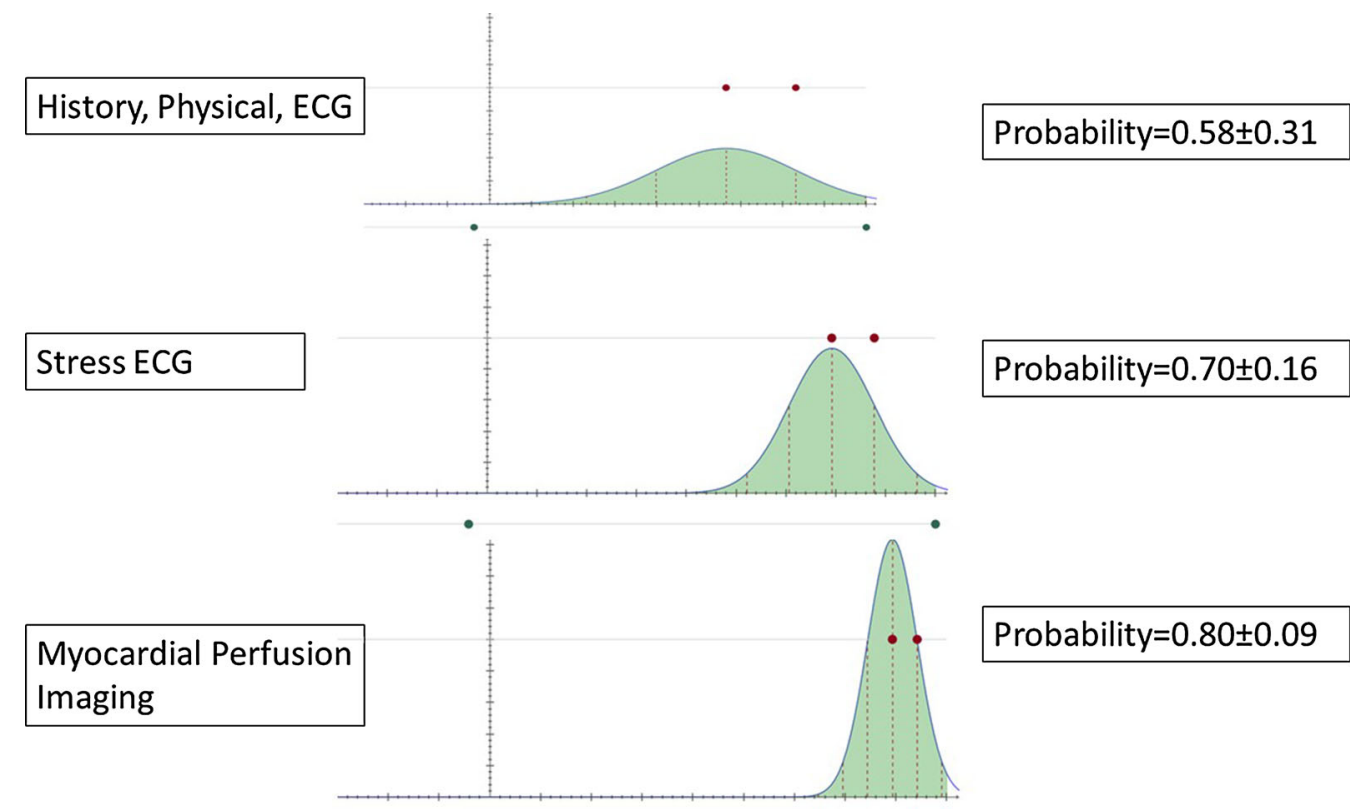

Figure 1. Cumulative probability curves at each stage of evaluation for a patient with chest pain. The starting probability is always $50 \%$. As history and physical data become available, the probability in this case increases to $58 \%$ but with wide confidence intervals. The patient remains at an intermediate risk of having disease. Subsequent testing (ECG stress) demonstrates an increased probability with narrower CI of the estimate but still remains in the intermediate risk range. Considering the clinical history and ECG stress results, the addition of perfusion imaging during stress puts the probability of CAD at $80 \%$ with tight confidence intervals of the estimate.

\section{Variability associated with each step in the serial evaluation of Imaging as a predictor of the response to CRT}

\section{Etiology of Dyssynchrony}
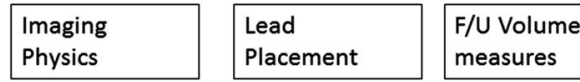
measures
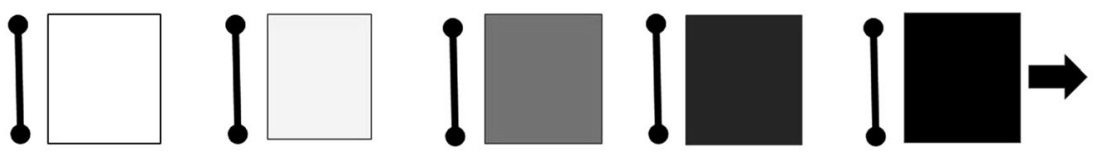

\section{Cumulative Variability Impact}

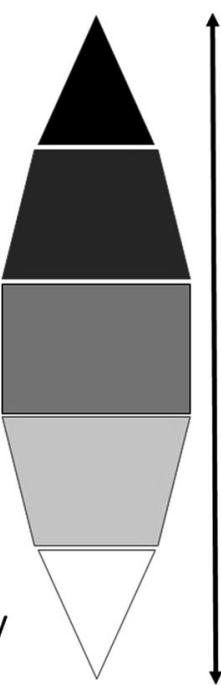

Figure 2. Schematic depiction of cumulative variability for assessing the impact of CRT based on imaging parameters. Each rectangle represents a degree of variability associated with each step of the evaluation. Arranged in series, they add cumulatively to generate an overall large amount of variability for the evaluation of any individual response to CRT. In addition to variability in the patient population inherent to the issue, there is sequential variability within the imaging modality (such as temporal resolution limits), the therapy itself, and the objective and subjective assessments of outcome. These are cumulative as the events occur in series. All of these are independent of the actual truth of the effectiveness of CRT therapy. 


\section{Disclosures}

The author has no relationships to disclose.

\section{References}

1. Petretta M, Petretta A, Cuocola A. Assessment of asynchrony by gated myocardial perfusion imaging improves patient management: Pro. J Nucl Cardiol. 2017. https://doi.org/10.1007/s12350-0171021-x.

2. Lee R, Shah RV, Murthy V. Assessment of dyssynchrony by gated myocardial perfusion imaging does not improve patient management. J Nucl Cardiol. 2017. https://doi.org/10.1007/s12350-0171022-9.

3. Zeitler EP, Friedman DJ, Daubert JP, Al-Khatib SM, Solomon SD, Biton Y, et al. Multiple comorbidities and response to cardiac resynchronization therapy: MADIT-CRT long-term follow-up. J Am Coll Cardiol 2017;69:2369-79.

4. Goldenberg Ilan, Moss Arthur J, Jackson Hall W, Foster Elyse, Goldberger Jeffrey J, Santucci Peter, et al. Predictors of response to cardiac resynchronization therapy in the multicenter automatic defibrillator implantation trial with cardiac resynchronization therapy (MADIT-CRT). Circulation 2011;124:1527-36.

5. Chung ES, Leon AR, Tavazzi L, et al. Results of the predictors of response to CRT (PROSPECT) trial. Circulation 2008;117:2608-16.

6. Malm S, Frigstad S, Sagberg E, Larsson H, Skjaerpe T. Accurate and reproducible measurement of left ventricular volume and ejection fraction by contrast echocardiography: a comparison with magnetic resonance imaging. J Am Coll Cardiol 2004;44:1030-5.

7. O'Keeffe S, Lye M, Donnellan C, Carmichael D. Reproducibility and responsiveness of quality of life assessment and six minute walk test in elderly heart failure patients. Heart 1998;80:377-82. 